\title{
Adaptative Markov Random Fields for Omnidirectional Vision
}

\author{
Cédric Demonceaux and Pascal Vasseur \\ C.R.E.A. (Centre de Robotique, d'Electrotechnique et d'Automatique) - EA 3299 \\ Université de Picardie Jules Verne \\ 7, Rue du Moulin Neuf \\ 80000 Amiens, France \\ Email: \{Cedric.Demonceaux, Pascal.Vasseur\}@u-picardie.fr
}

\begin{abstract}
Images obtained with catadioptric sensors contain significant deformations which prevent the direct use of classical image treatments. Thus, Markov Random Fields (MRF) whose usefulness is now obvious for projective image processing, can not be used directly on catadioptric images because of the inadequacy of the neighborhood. In this paper, we propose to define a new neighborhood for MRF by using the equivalence theorem developed for central catadioptric sensors. We show the importance of this adaptation for a motion detection application.
\end{abstract}

\section{Introduction}

The interesting properties of omnidirectional vision has led to the development of different acquisition systems such a camera networks, rotating cameras or catadioptric sensors [3]. Catadioptric vision consists in associating a convex mirror with a projective camera whose the optical axis is aligned with the axis of the mirror (Fig. 1). The main advantage of these sensors is the acquisition of an omnidirectional image with a single shot. However, catadioptric image contains significant deformations due to the geometry of the mirror and to the sampling of the camera [5](Fig. 1). These deformations have important consequences for the image processing and the direct application of classical operators can not provide satisfactory results.

Thus, although, since the eighties, MRF have permitted to solve many problems in image processing such as image segmentation, image restauration, motion detection and so on, they become unsuitable if they are applied in the same way for catadioptric images. Indeed, MRF use a local dependence between pixels from a predefined neighborhood which has to be reconsidered because of the deformations of the catadioptric image.

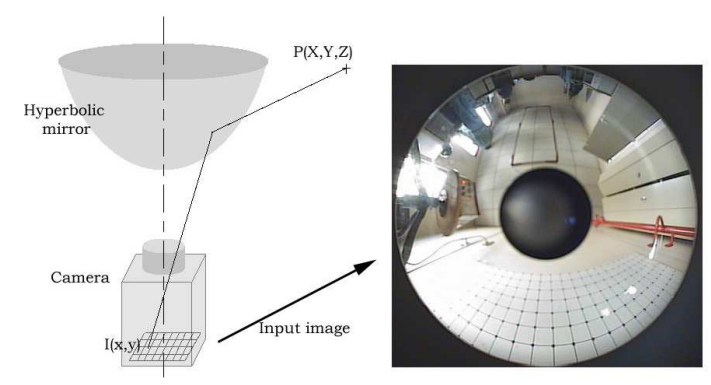

Figure 1. Omnidirectional sensor and omnidirectional image.

The aim of this paper is then to propose an adaptation of the MRF for the catadioptric images from the definition of a new neighborhood system which takes into account the deformations. We define this neighborhood from the equivalence between the catadioptric image formation and the projection of the $3 \mathrm{D}$ points on the image plane via a unitary sphere [7].

\section{Markov Random Fields in Image Processing}

Spatial MRF have been introduced by Besag [4] and popularized in image processing by Geman and Geman [6]. These models are defined from a graph $\mathcal{G}=(\mathcal{S}, \mathcal{U})$ where $\mathcal{S}=\left\{s_{1}, s_{2}, \cdots, s_{N}\right\}$ is the set of vertices (named set of sites in the case of MRF) and $\mathcal{U}$ the set of edges. In this graph $\mathcal{G}$, two sites $s_{i}$ and $s_{j}$ are neighbors if there exists an edge $u_{i j} \in \mathcal{U}$ between them. Thus, the set of neighbors of a site $s$ is noted $\mathcal{V}_{s}$.

From $\mathcal{V}=\left\{\mathcal{V}_{s} \mid s \in \mathcal{S}\right\}$, we define the set $\mathcal{C}$ whose elements 
$c$ (Fig 2), named cliques, are the parts of $\mathcal{S}$ which verify

$$
\left\{\begin{array}{c}
\exists s \in \mathcal{S} \text { such as } c=\{s\} \\
\text { or } \\
\forall\{s, t\} \subset c, \quad t \in \mathcal{V}_{s}
\end{array}\right.
$$

Consider a random field $\mathcal{X}=\left\{X_{1}, \cdots, X_{N}\right\}$ of realization $\omega=\left\{\omega_{1}, \cdots, \omega_{N}\right\}$

Definition $1 \mathcal{X}$ is a MRF according to $\mathcal{V}$ if :

$$
\begin{array}{lll}
\text { (i) } \quad \forall \omega & P(\mathcal{X}=\omega)>0 \\
\text { (ii) } & \forall(s, \omega) & P\left(X_{s}=\omega_{s} \mid X_{r}=\omega_{r}, r \neq s\right)= \\
& P\left(X_{s}=\omega_{s} \mid X_{r}=\omega_{r}, r \in \mathcal{V}_{s}\right)
\end{array}
$$

A Markov field is a random field where each site is only influenced by its neighboring sites. Using the characterization (1) does not permit to obtain a simple expression of joint distribution $P(\mathcal{X}=\omega)$. However, this distribution can be easily obtained from the Hammersley-Clifford theorem [4] which establishes the equivalence between the Markov fields and the Gibbs fields.

Problems such as image restauration, classification and segmentation can be schematized as follows: from observations $Y$ (known information) in the image or in the sequence (grey level, color, edges, displacements, ...), we want to find the set of labels $X$ (hidden information) which could originate these observations. The solution of these problems requires to clarify the relation between the labels and the sites. However, these models are not sufficient to solve these inverse problems. Indeed, because of the loss of information during the image formation and because of the noise in the observations, these inverse problems are generally ill-posed according to Hadamard. We have to make an additional hypothesis on the researched set of labels. This is why we add a constraint named a priori knowledge which forces the set of labels to be Markovian.

The bayesian estimation permits to modelize jointly the labels and the observations thanks to the coupled random field $(X, Y)$ with the probability distribution $P_{X Y}$. For example, if we consider the bayesian estimator Maximum $a$ Posteriori (MAP), the researched markovian field of labels $x$ is solution of

$$
\arg \min _{x \in \Omega} U(x, y)=\arg \min _{x \in \Omega}\left(U_{1}(x, y)+U_{2}(x)\right)
$$

where $U_{1}$ is the term linked to the data which expresses the relation between observations $y$ and labels $x$ and where $U_{2}$ describes the markovian a priori of the label field and is decomposed into a sum of local potentials which depends on the neighboring system:

$$
U_{2}(x)=\sum_{<s, s^{\prime}>\in \mathcal{C}} V_{2}\left(s, s^{\prime}\right) .
$$

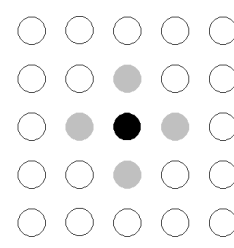

(a)

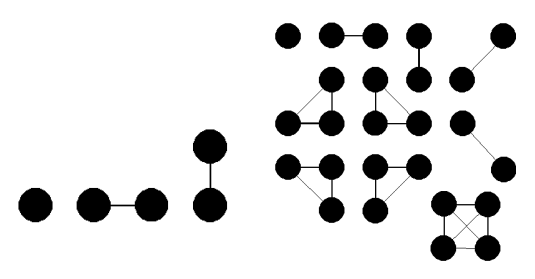

(c)

\section{Figure 2. Neighboring system and associated cliques. Neighborhood (a) and cliques (c) of first order, Neighborhood (b) and cliques (d) of second order.}

In the case of classical images, the neighboring systems are defined by the closest pixels (Fig 2). However, this kind of neighborhood is not suited to catadioptric images because of the deformations generated by the mirror. Indeed, a pixel at a distance $d$ of pixel $p$ does not have the same influence on the latter if $p$ is placed either in the center of the image or in the periphery (Fig 3).

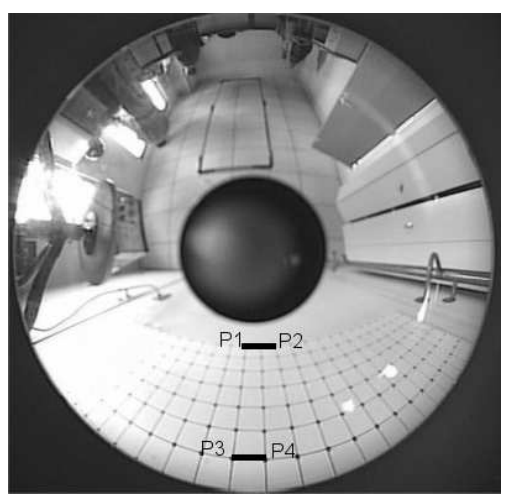

Figure 3. $d(P 1, P 2)=d(P 3, P 4)$ when $P 1$ does not have physically the same influence on $P 2$ than $P 3$ on $P 4$. 


\section{Central Catadioptric MRF}

\subsection{Catadioptric Image Formation and Equivalence Theorem}

Baker and Nayar classified catadioptric sensors into two categories according to the number of viewpoint [1]. Thus, sensors with a single viewpoint, named central catadioptric sensors, permit a geometrically correct reconstruction of the perspective image from the original catadioptric image. This category is made of parabolic mirror associated to orthographic camera and hyperbolic, elliptic and plane mirrors with perspective camera. The second category with several viewpoints has geometric properties very less significant and is made of the other possibilities of association between mirrors and cameras. In this paper, we are only interested in central sensors for which Geyer and Daniilidis have demonstrated the equivalence with a two-step projection via a unitary sphere centered on the focus of the mirror (the single viewpoint) [7]. This two-step projection consists first in projecting a $3 \mathrm{D}$ point to the sphere from the center of the sphere. The next step consists in projecting the point on the sphere to the image plane from a point placed on the optical axis (Fig 4). The positions of this point of projection and of the image plane are determined with the calibration of the sensor.

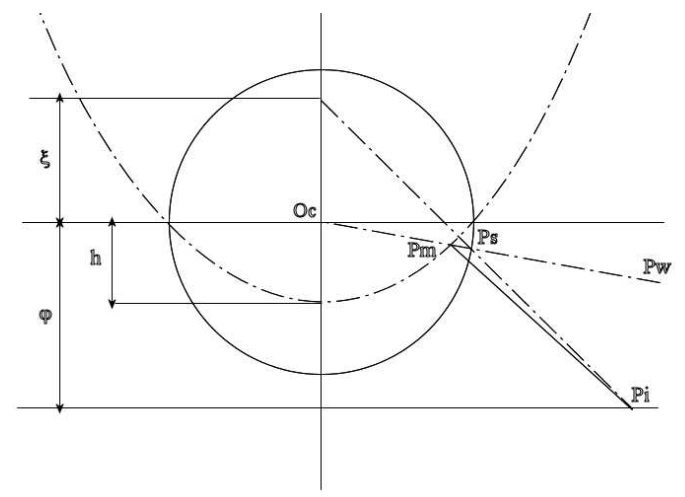

Figure 4. Equivalence between the catadioptric projection and the two-step mapping via the sphere.

This equivalence is very interesting since it allows to perform image processing in a new space in which the deformations are taken into account. Many authors used this space in order to compute the derivative of catadioptric image [5], the pose of the sensor [9] or to detect catadioptric lines [2]. We also propose to use this space in order to determine the most appropriate neighboring system for the central catadioptric MRF.

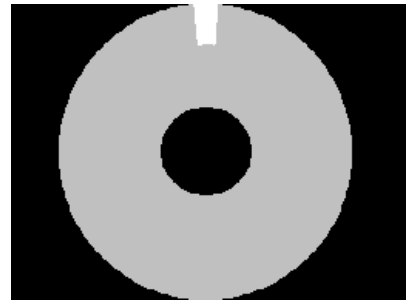

(a)

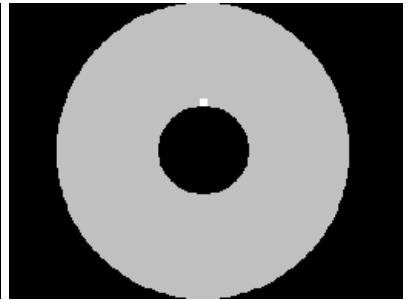

(b)
Figure 5. (a) Neighborhood at the periphery of the image, (b) Neighborhood in the center of the image.

\subsection{Central Catadioptric Neighboring System}

Let $\mathrm{P}$ the projection for which any point $P_{i}$ in the catadioptric image associates its equivalent point $P_{s}$ in spherical coordinates on the unitary sphere $S^{2}$ (Fig 4),

$$
\mathrm{P}: \begin{array}{rlc}
\mathcal{S} & \rightarrow & S^{2} \\
s & \mapsto\left(1, \theta_{s}, \phi_{s}\right)
\end{array}
$$

Then, we define the new neighborhood as follows:

$$
\begin{aligned}
& \forall s \in \mathcal{S}, \mathrm{P}(s)=\left(1, \theta_{s}, \phi_{s}\right) \\
& \mathcal{V}_{s}=\left\{\begin{array}{l}
t \in \mathcal{S}, t \neq s \quad \mathrm{P}(t)=\left(1, \theta_{t}, \phi_{t}\right) \text { such as } \\
\left|\theta_{t}-\theta_{s}\right| \leq \frac{1}{N} \text { and } \\
\left(\left|\phi_{t}-\phi_{s}\right| \leq \frac{1}{M} \text { or }\left|\phi_{t}-\phi_{s}\right| \geq 2 \pi-\frac{1}{M}\right)
\end{array}\right\},
\end{aligned}
$$

\section{Experimentation}

In this part, we present results obtained from a markovian modelling adapted to catadioptric images in the case of motion detection.

Consider two consecutive images $I(t)$ and $I(t+1)$ from a sequence acquired from a fixed catadioptric sensor. The problem consists in detecting the moving parts in the image between the instants $t$ and $t+1$. For this, we pose $E=$ $\left\{e_{s}, s \in \mathcal{S}\right\}$ the label field where $e_{s}$ have values in $\{0,1\}$ $\left(0=\right.$ motionless site, $1=$ mobile site) and $O=\left\{o_{s}, s \in \mathcal{S}\right\}$ is the observation field. We consider the following markovian energy:

$$
U(e, o)=\sum_{s \in \mathcal{S}} f\left(e_{s}, o_{s}\right)+\lambda \sum_{<s, s^{\prime}>\in \mathcal{C}}\left(1-\delta\left(e_{s}, e_{s}^{\prime}\right)\right)
$$




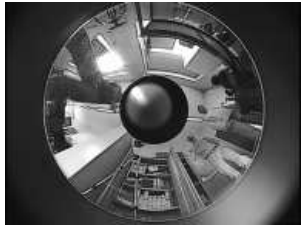

(a)

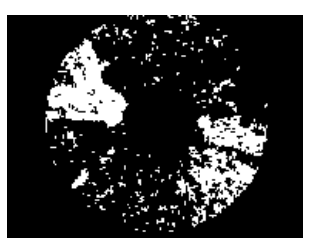

(c)

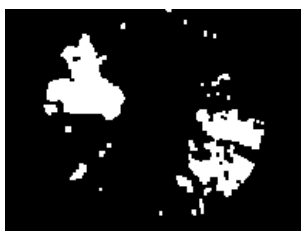

(e)

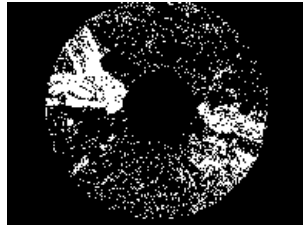

(b)

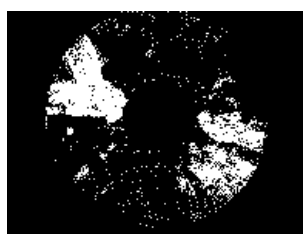

(d)

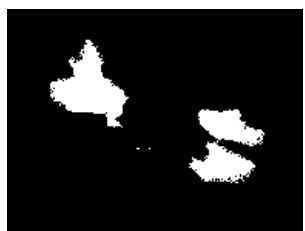

(f)
Figure 6. (a) Image at instant $t$, (b) Maximum of likelihood, (c) classical markov $\lambda=0.6$,(d) catadioptric markov $\lambda=0.6$, (e) classical markov $\lambda=1.2,(\mathbf{d})$ catadioptric markov $\lambda=$ 1.2 .

where

$f\left(e_{s}, o_{s}\right)=\left\{\begin{array}{cl}1-\frac{1}{1+\exp (|I(s, t)-I(s, t+1)|-\alpha)} & \text { if } e_{s}=0 \\ \frac{1}{1+\exp (|I(s, t)-I(s, t+1)|-\alpha)} & \text { if } e_{s}=1\end{array}\right.$

and

$$
\delta(x, y)= \begin{cases}1 & \text { if } x=y \\ 0 & \text { if } x \neq y\end{cases}
$$

We have compared our markovian modelling adapted to the central catadioptric images with a modelling obtained from a classical neighborhood of first order (Fig 2(a)). Constants $N$ and $M$ are chosen in order to obtain a catadioptric neighborhood similar than the first order neighborhood in the center of the image. We set $\alpha=3$ (threshold from which we consider the pixels in motion). Between instants $t$ and $t+1$, three persons are moving in front of the camera. Maximum of likelihood given in Fig. (6) is minimized by the deterministic algorithm I.C.M. [6] with two values of $\lambda$. If $\lambda=0.6$, the dependence between the neighbors is not sufficient in order to remove the noise. With $\lambda=1.2$, the catadioptric neighborhood allows to remove all the false positive in contrary to the classical neighborhood. In the latter case, we can also note that a block effect appears in the results.

\section{Conclusion and perspectives}

In this paper, we have proposed an adaptation of the markovian modelling to the central catadioptric images. Experimentations show very encouraging results. In the same way than Heitz et al [8] for the classical case, it should be very interesting to develop a multi-scaling method in order to reduce the sensitivity of the initialization of the deterministic algorithms and to speed-up the treatment.

\section{Acknowledgment}

This work is supported by Agence Nationale de la Recherche - projet CAVIAR (ANR-05-BLAN-0291-01).

\section{References}

[1] S. Baker and S.K. Nayar. A theory of single-viewpoint catadioptric image formation. Int. Journal on Computer Vision, 35(2):175-196, November 1999.

[2] J.P. Barreto and H. Araujo. Direct least square fitting of paracatadioptric line images. In Proc. Workshop on Omnidirectional Vision and Camera Networks, OMNIVISO3, Madison, Wisconsin, June 2003.

[3] R. Benosman and S.B. Kang. Panoramic Vision: Sensors, Theory, Applications. Springer, 2001.

[4] J. Besag. Spatial interaction and the statistical analysis of lattice systems. RoyalStat, B-36(2):192-236, 1974.

[5] K. Daniilidis, A. Makadia, and T. Blow. Image processing in catadioptric planes: Spatiotemporal derivatives and optical flow computation. In Proc. Workshop on Omnidirectional Vision, OMNIVISO2, pages 3-10, Copenhagen, Denmark, June 2002.

[6] S. Geman and D. Geman. Stochastic relaxation, gibbs distributions, and the bayesian restoration of images. IEEE Trans. on Pattern Analysis and Machine Intelligence, 6(6):721-741, November 1984.

[7] C. Geyer and K. Daniilidis. Catadioptric projective geometry. Int. Jounal on Computer Vision, 45(3):223243, December 2001.

[8] F. Heitz, P. Perez, and P. Bouthemy. Multiscale minimization of global energy functions in some visual recovery problems. CVGIP: Image Understanding, 59(1):125-134, January 1994.

[9] A. Paulino and H. Araújo. Pose estimation for central catadioptric systems: An analytical approach. In Proc. Int. Conf. on Pattern Recognition, ICPR02, pages 696699, Québec, Canada, August 2002. 